\title{
Fourier analyses of water-reinforced response rates at two levels of thirst in the rat
}

\author{
LOWELL T. CROW \\ Western Washington University, Bellingham, Washington
}

\begin{abstract}
Rats were trained under a 24-h water-deprivation regimen to press either of two bars to obtain water reinforcement in a daily 32 -min operant session, then tested after a 47 -h water-deprivation period. Measures were made of uncertainty of left- or right-bar response sequences and of the Fourier frequency spectra of 30-sec response rates throughout each session. Coefficients of variation of frequency magnitudes, but not uncertainty values, were significantly reduced by increased thrist.
\end{abstract}

It has been suggested (Crow, 1977) that behavioral variability may be related to "environmental tolerance" and to the functional complexity of the nervous system. For example, the imposition of reinforcement contingencies reduce behavioral variability, the relaxation of these contingencies (extinction) increases behavioral variability, and several kinds of brain lesions and drug effects reduce behavioral variability (see Crow, 1985, for review). Indices of behavioral variability have included uncertainty scores derived from multiple response sequences and, more recently, Fourier analysis of response rates. In an earlier study of alcohol effects (Crow, 1990), the variability within the frequency spectra of response rates was found to be a sensitive measure of operantresponse-rate changes. The present study was designed to further compare these two assessments of behavioral variability, uncertainty scores, and Fourier analysis in a different, unexplored area-that of the effects of drive on behavioral variability.

\section{METHOD}

\section{Subjects}

Ten experimentally naive male Sprague-Dawley albino rats were used as subjects. They were approximately 90 days of age at the beginning of the experiment.

\begin{abstract}
Apparatus
Two Gerbrands two-bar Skinner boxes, modified for water reinforcement, were used in conjunction with two Atari $800 \mathrm{XL}$ personal computers to program reinforcement and collect response-rate data.

\section{Procedure}

The subjects were put on a 24-h water-deprivation regimen for 1 week, after which they were trained to barpress for water reinforcement in two-bar operant chambers. A 15-min free-drinking period always followed the daily 32-min operant session. The rats were first autoshaped for 2 days with a program that provided reinforcement every $30 \mathrm{sec}$ regardless of the animal's response but that reinforced presses on either bar. On the 3rd day, all animals were put, without manual shaping, on
\end{abstract}

Correspondence should be addressed to Lowell T. Crow, Department of Psychology, Western Washington University, Bellingham, WA 98225-5996. continuous reinforcement (either bar providing reinforcement) and kept on continuous reinforcement for the remainder of the experiment. When responding rates had stabilized, 18 successive days after the beginning of autoshaping, all animals were subjected to a 47-h water-deprivation period (one daily session of drinking was skipped), then tested in the operant chamber.

\section{RESULTS}

The left panels of Figures 1 and 2 show the mean response rates for the 10 animals at the 30 -sec intervals of the relevant 32-min operant session, the 24-h deprivation condition (D24), or the 47-h deprivation condition (D47).

Related $t$ tests revealed a significantly greater mean number of responses for D47 (111.7) relative to D24 $(100.5)[t(9)=2.938]$. Although there was a greater mean uncertainty score (Frick \& Miller, 1951) for condition D24 (0.353) relative to D47 $(0.313)$, there was no significant difference between these means $[t(9)=0.590]$, which represented the variability of left- and rightbarpresses. Either bar provided reinforcement; each animal usually adopted a more preferred bar that did not appear to vary with conditions.

Using total responses for each 30-sec period of the 32min sessions, 64-point fast Fourier transforms (FFTs)
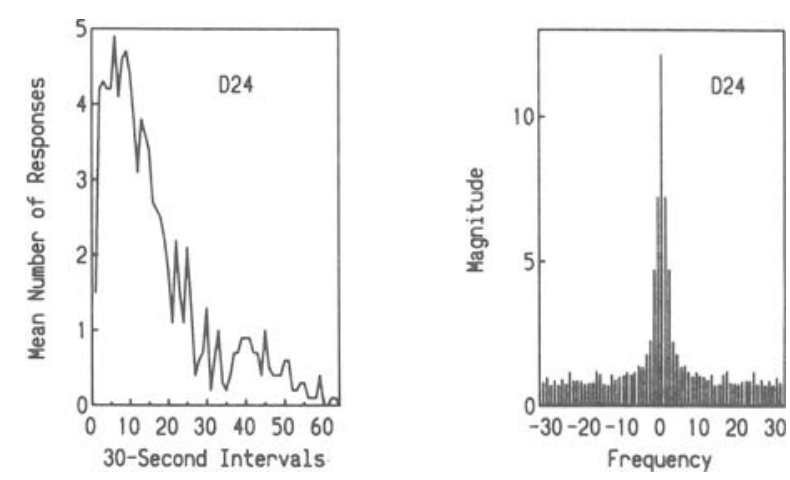

Figure 1. Mean response rates and mean frequency magnitudes for the 24-h deprivation condition. 

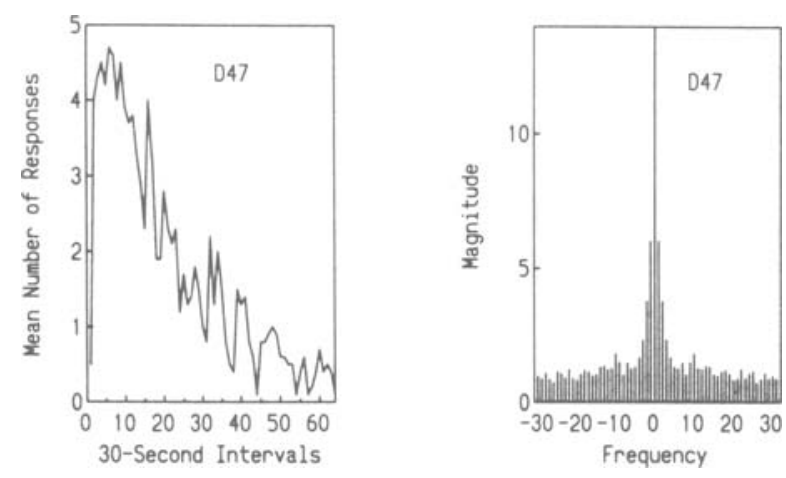

Figure 2. Mean response rates and frequency magnitudes for the 47-h deprivation condition.

were computed for each animal in each session under each deprivation condition. The frequency spectra are summarized in the right panels of Figure 1. These summaries were obtained by averaging each frequency magnitude for the 10 animals. Thus, each spike in the figures is based on 10 FFTs. The magnitude represents the relative extent to which a frequency is a part of the overall timebased function of responses per $32 \mathrm{~min}$. The transform of the time-based data into the frequency domain represents response-rate "cycles" within the 32-min operant session. Thus, Frequency 1 represents one cycle per $32 \mathrm{~min}$, Frequency 2 represents two cycles per $32 \mathrm{~min}$ or one cycle per $16 \mathrm{~min}$, and so on. Both positive and negative frequencies make up the sine-wave/cosine-wave approximation of the time-based function.

Although the frequency spectra of mean magnitudes for D24 and D47 are similar and look, to an extent, like onehalf sine waves, there are differences in the two. For each FFT, a coefficient of variation (CV, standard deviation/mean) was obtained for the 64 points for each FFT. Thus, there was one CV for each animal for each condition representing the extent of variation within the frequency magnitudes. Related $t$ tests showed a higher mean CV for the 64 points of the D24 condition than for those of the D47 condition $[t(9)=2.368]$. There was also a significant mean difference in CVs in the same direction using the 32 points of positive frequencies only $[t(9)=$ 3.772]. There were significant mean magnitude differences at Frequency $10[t(9)=0.302]$, indicating an increase in response-rate cycles of $3.1 \mathrm{~min}$ for the D47 condition.

\section{DISCUSSION}

As with earlier work, the present study indicates significant differences in behavioral variability in expected directions by use of the Fourier frequency spectra of the response rates when uncertainty values do not discriminate between conditions. For example, it was found that variability contingent behaviors were affected by alcohol only if Fourier analyses were considered (Crow, 1990) and that response-sequence analysis may assess an aspect of behavioral variability different from that assessed by response-rate analysis.

The uncertainty index has been a valuable aid to the study of behavior variability, but there appears to be more to behavioral variability than randomness. There is, in addition, the occurrence or nonoccurrence of particular responses within a specific time frame (response rate or frequency) and the pattern of change of response rates over time (accelerations or decelerations of rate). The transformation of time-based data into the frequency domain, the forward Fourier transform, allows for a unique assessment of behavioral variability and one that appears to be more sensitive and reliable than informational analysis of response orders.

The patterns in responses related to the relief of thirst revealed by Fourier transforms may represent underlying physiological satiety processes of gastrointestinal filling and absorption. For example, there appears to be a basic cycle of approximately $3.1 \mathrm{~min}$ that could relate to stomach emptying.

\section{REFERENCES}

Crow, L. T. (1977). Is variability a unifying behavioral concept? Psychological Record, 27, 783-790.

Crow, L. T. (1985). More on variability as a behavioral concept. Psychological Record, 35, 252-300.

Crow, L. T. (1990). Alcohol effects on operant-rate frequency spectra are schedule-dependent. Bulletin of the Psychonomic Society, 28, 445-447.

FrICK, F. C., \& MilLer, G. A. (1951). A statistical description of operant conditioning. American Journal of Psychology, 64, 20-36.

(Manuscript received March 25, 1991.) 